\title{
Human umbilical cord blood cells restore vascular integrity in injured rat brain and modulate inflammation in vitro
}

\author{
Amit K Srivastava ${ }^{1}$, Karthik S Prabhakara ${ }^{1}$, Daniel J Kota ${ }^{2}$, Supinder S Bedi ${ }^{1}$, Fabio Triolo ${ }^{1}$, \\ Katherine S Brown ${ }^{3}$, Matthew L Skiles ${ }^{3}$, Heather L Brown ${ }^{3}$, Charles S Cox Jr ${ }^{1}$ \& Scott D \\ Olson*,1 \\ ${ }^{1}$ Department of Pediatric Surgery, McGovern Medical School, University of Texas Health Sciences Center at Houston, Houston, \\ TX 77030, USA \\ ${ }^{2}$ Emory Personalized Immunotherapy Core Labs, Emory University, School of Medicine, Atlanta, GA 30322, USA \\ ${ }^{3}$ Cbr Systems, Inc., South San Francisco, CA 94080, USA \\ *Author for correspondence: Tel.: +1 713500 7259; scott.d.olson@uth.tmc.edu
}

\begin{abstract}
Aim: Traumatic brain injury is a complex condition consisting of a mechanical injury with neurovascular disruption and inflammation with limited clinical interventions available. A growing number of studies report systemic delivery of human umbilical cord blood (HUCB) as a therapy for neural injuries. Materials \& methods: HUCB cells from five donors were tested to improve blood-brain barrier integrity in a traumatic brain injury rat model at a dose of $2.5 \times 10^{7}$ cells/kg at 24 or $72 \mathrm{~h}$ postinjury and for immunomodulatory activity in vitro. Results \& Conclusion: We observed that cells delivered $72 \mathrm{~h}$ postinjury significantly restored blood-brain barrier integrity. HUCB cells reduced the amount of TNF- $\alpha$ and IFN- $\gamma$ released by activated primary rat splenocytes, which correlated with the expression of COX2 and IDO1.
\end{abstract}

Representative coronal sections from experimental groups injured with a sham injury (top left), a controlled cortical impact (top right), or a controlled cortical impact and an infusion with human umbilical cord blood cells (bottom left) $72 \mathrm{~h}$ after injury and imaged following intravenous infusion of a large farred fluorescent dye to visualize blood-brain barrier leakage at $96 \mathrm{~h}$ after injury. This technique is used to quantitatively measure vascular permeability as a biomarker for injury (bottom right).

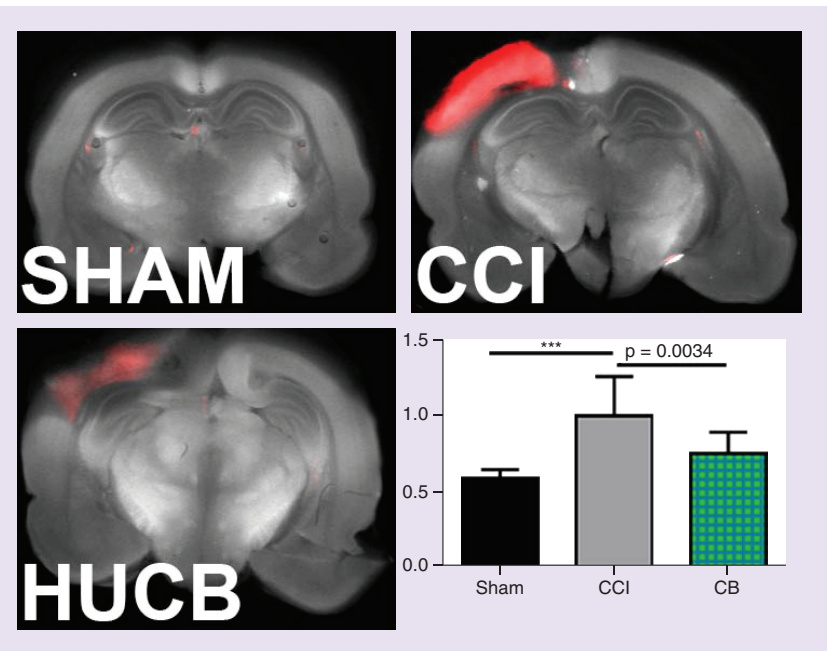

First draft submitted: 22 August 2018; Accepted for publication: 1 March 2019; Published online: 10 May 2019

Keywords: blood-brain barrier • cell therapy • cord blood • immunomodulation • inflammation 
Each year approximately $1 \%$ of the population of developed countries sustain some form of brain injury [1] and those injuries become one of the major causes of death, especially in young adults [2]. Developing relevant therapies for brain injuries has proven to be challenging, particularly due to the insidious, progressive neurodegenerative course of the disease. Cell damage, right after the primary insult to the brain, is irreversible and not amenable to intervention. This primary injury triggers a cascade of molecular events that leads to a secondary injury causing further neuronal damage [3]. Because restorative therapies targeting the underlying cause of brain injury do not exist, stem cell therapies with anti-inflammatory, neuroprotective and regenerative potential hold great promise. In the last decade, several studies showed administration of progenitor stem cells as a promising tissue repair strategy in an injured brain [3-9]. These cells improve the vascular microenvironment, promote axonal growth and exert anti-inflammatory effects [10].

Human umbilical cord blood (HUCB), which is readily available at birth, is a rich source for progenitor stem cells at different lineage commitment stages (hematopoietic, endothelial, mesenchymal and neural progenitor stem cells) [11]. Cord blood is an accepted graft source for adult and pediatric patients in need of a hematopoietic stem cell transplant and the use of cord blood-derived cells, outside the setting of hematological disease, is an active area of investigation in various regenerative medicine applications [12]. Among the advantages of using HUCB cells are that it can be collected in a noninvasive manner and it lacks the ethical considerations associated with research utilizing other stem cell sources [13]. HUCB units can be stored in public banks where they are made available to suitably matched, unrelated patients in need of a stem cell transplant as well as private banks for autologous transplantation or as a donor source for first and second degree relatives [14]. In addition, due to higher number of immature progenitor cells, and weak immunogenicity [15], HUCB requires less stringent human leukocyte antigen (HLA) matching compared with bone marrow, extending the option for transplantation to patients who lack a sufficiently matched sibling or bone marrow registry donor. Fewer HLA disparities between unit and recipient result in lower transplant-related mortality and improved overall survival [16-18] and make these cells an accepted alternative to bone marrow-derived cells for transplantation.

In addition to well-established applications in hematopoietic stem cell transplantation, these cells have the potential to facilitate tissue repair in the CNS [19-22]. HUCB cells have also demonstrated the capacity to modulate immune and inflammatory reactions at cellular and molecular levels [23-26] and early-stage clinical trials point to the safety of administering autologous HUCB cells in various neurological injuries [27,28]. However, despite several preclinical and clinical studies, little is known about the mechanism of HUCB cell-mediated immunomodulation.

To elucidate the therapeutic potential of HUCB cells, we utilized an experimental rat model of traumatic brain injury (TBI). Neuroinflammation is salient part of the TBI pathophysiology $[29,30]$ and blood-brain barrier (BBB) disruption, a pathological hallmark of TBI, can be both a cause and an effect of CNS inflammation [31]. We evaluated whether systemic administration of HUCB cells restores BBB integrity in experimental TBI. We further investigated the immunomodulatory abilities of HUCB cells in vitro and the underlying mechanisms.

\section{Materials \& methods}

\section{Cord blood harvesting \& processing}

For this study, five HUCB units (CB-1, CB-2, CB-3, CB-4 and CB-5; see Table 1) were utilized. Informed written consent was obtained from donors prior to delivery and all HUCB collections were from full-term births (defined as $>37$ weeks gestation, based on self-reported data). The collection of HUCB was exempted from Institutional Review Board approval. Following delivery, HUCB was collected into gravity bags containing 500 units of lyophilized heparin. HUCB was then transported to Cord Blood Registry processing facility in Tucson, Arizona, and volume and red blood cell reduced on automated and a closed AutoXpress ${ }^{\circledR}$ Platform (Cesca Therapeutics, Inc., CA, USA) system. The resulting product was then transferred to a storage bag with a major and minor compartment representing 80 and 20\% volumes, respectively. All research presented here were performed using the major compartment.

All the cord blood units were stored at $-80^{\circ} \mathrm{C}$ upon arrival and were thawed in a $37^{\circ} \mathrm{C}$ water bath until sample reaches a slushy/liquid consistency. Following processing, $1 \mathrm{ml}$ of product was removed and assessed for hematologic parameters including total nucleated cell (TNC) and mononuclear cell (MNC) counts on a Sysmex hematology analyzer (model XE-2100 L, Sysmex America, IL, USA; Table 1), and viability was determined by propidium iodide and acridine orange or 7-AAD staining. Cord blood units were cryopreserved in a $10 \%$ dimethyl sulfoxide/1\% 
Table 1. Human umbilical cord blood unit information. Collected cords were anonymized prior to delivery by a third party. The collection volume, viability prior to cryopreservation, adjusted total nucleated cell and mononuclear cell counts, as well as the total nucleated cell fraction present in the 'major' compartment.

\begin{tabular}{|c|c|c|c|c|c|}
\hline Unit ID & Collection volume (ml) & $\begin{array}{l}\text { Postprocessing viability } \\
\text { (\%) }\end{array}$ & $\begin{array}{l}\text { Postprocessing viable } \\
\text { TNC }\left(10^{6}\right)\end{array}$ & Postprocessing MNC $\left(10^{6}\right)$ & $\begin{array}{l}\text { Major compartment viable } \\
\text { TNC }\left(10^{6}\right)\end{array}$ \\
\hline CB-2 & 65.95 & 85.4 & 1550.74 & 431.18 & 1195.62 \\
\hline CB-4 & 128.97 & 98.77 & 1394.43 & 669.07 & 1075.11 \\
\hline CB-5 & 66.38 & 98.93 & 1083.4 & 325.83 & 903.84 \\
\hline
\end{tabular}

MNC: Mononuclear cell; TNC: Total nucleated cell.

dextran 40 solution (final concentration; VWR International, PA, USA) using a microprocessor-controlled rate freezer (Custom Biogenic Systems, MI, USA) with final storage in a cryogenic dewar (MVE, Pacific Science, CA, USA) at $-196^{\circ} \mathrm{C}$ in the vapor phase of liquid nitrogen. All cord blood units were processed within $48 \mathrm{~h}$ of collection and were determined to be free of aerobic or anaerobic microbial contamination (BacT ALERT ${ }^{\circledR}$ 3D system, bioMerieux, Marcy-l'Étoile, France). Cord blood storage bags were inspected for integrity upon retrieval and shipped overnight on dry ice with a temperature-monitoring device (LogTag ${ }^{\circledR}$ TRIL-8, MicroDAQ, NH, USA). Prior to infusion, cells were suspended in phosphate-buffered saline (PBS) at a concentration of $2.5 \times 10^{7}$ cells $/ \mathrm{kg}$ (final volume: $1 \mathrm{ml}$ ). Throughout the experiment, we used parameters selected to be consistent with real-world usage scenarios.

\section{Controlled cortical impact rat model}

All animal experiments were approved by the institutional Animal Welfare Committee of the University of Texas Health Science Center at Houston (TX, USA) in compliance with the NIH Guide for the Care and Use of Laboratory Animals.

For this study, we used an electromagnetic controlled cortical impact (CCI) device (Impact One Stereotaxic Impactor, Leica Microsystems, IL, USA) to administer a unilateral mechanical brain injury in rats as described previously [32]. Use of this device produces cortical contusion, disruption of $\mathrm{BBB}$, hippocampal cell loss and overall brain volume loss [33].

In brief, male Sprague-Dawley rats weighing 250-275 g were anesthetized with $4 \%$ isoflurane. The heads of rats were then mounted in a stereotactic frame and held in a horizontal plane. A midline incision was made and a 6-7 mm diameter craniectomy was performed on the right cranial vault. The center of the craniectomy was placed at the midpoint between bregma and lambda, $3 \mathrm{~mm}$ lateral to the midline, overlying the temporoparietal cortex. Rats received $3.1 \mathrm{~mm}$ depth of deformation with an impact velocity of $5.8 \mathrm{~m} / \mathrm{s}$ and a dwell time of $150 \mathrm{~ms}$ (moderate-severe injury) to the parietal association cortex orthogonal to the surface and at an angle of $10^{\circ}$ from the vertical plane. Sham injury was performed making a midline incision and separating the skin, connective tissue and aponeurosis from the cranium. Incisions in both the sham operated and injury groups were closed using skin clips.

\section{Cell transplantation}

A total of 60 rats were used in this study. Animals were divided into the following groups: $\mathrm{CCI}$ injury alone $(\mathrm{n}=10)$; CCI with CB-1 (cells delivered $24 \mathrm{~h}$ after the injury, $\mathrm{n}=10$ ); CCI with CB-1 (cells delivered $72 \mathrm{~h}$ after the injury, $\mathrm{n}=10$ ); CCI with CB-2 (cells delivered $24 \mathrm{~h}$ after the injury, $\mathrm{n}=10$ ); CCI with CB-2 (cells delivered $72 \mathrm{~h}$ after the injury, $\mathrm{n}=10)$; and sham $(\mathrm{n}=10)$. Prior to cell injection, cell viability was determined and cells were suspended in PBS (final volume: $1 \mathrm{ml}$ ) for intravenous administration via tail vein at the dose of $2.5 \times 10^{7}$ cells $/ \mathrm{kg}$ bodyweight. CCI alone group received vehicle (PBS) and sham group only received an incision.

\section{BBB permeability measurement}

CCI rats that received cells at $24 \mathrm{~h}$ after the injury were analyzed for BBB permeability at 3 days postinjury and rats that received cells at $72 \mathrm{~h}$ after the injury were analyzed for BBB permeability at 4 days postinjury. BBB permeability was measured using a far red dye (Alexa Fluor 680, ThermoFisher Scientific, MA, USA) bioconjugated to a $10 \mathrm{kDa}$ dextran molecule as previously reported by our group [34] and has been used in several additional studies [32,35,36]. 
In brief, 30 min prior to euthanasia, rats were intravenously injected with $1 \mathrm{ml}$ of $1 \mathrm{mg} / \mathrm{ml}$ Alexa Fluor 680 dye. Following exsanguination and 4\% paraformaldehyde cardiac perfusion, the brains were harvested, sliced in $2 \mathrm{~mm}$ coronal sections and scanned for Alexa Fluor 680 signal using an Odyssey laser scanner (LI-COR Biosciences, NE, USA) in the $700 \mathrm{~nm}$ emission channel while autofluorescence was visualized in the nonspecific $800 \mathrm{~nm}$ emission channel. A composite image was created for visualization purposes by adjusting the brightness and contrast to accommodate the minimum and maximum intensity of the images and then overlayed by assigning the Alexa Fluor 680 a red color and the nonspecific fluorescence in the 800 channel a gray color. The signal intensity (amount of dye accumulated) within the whole brain was calculated relative to sham rat brain. For the signal of intensity calculation, regions of interest were created of uniform area to encompass the entirety of $62 \mathrm{~mm}$ slices from each brain within a single image. The mean fluorescence and area of each region of interest was then measured using a threshold set to exclude intensities below 1000 from analysis to remove background and autofluorescence. The values were normalized to the sham controls from each cohort (two cohorts were used for the different time courses).

\section{Primary splenocyte inhibition/activation assay}

Sprague Dawley rats were purchased from Harlan (IN, USA) and splenocytes were isolated from those rats as described previously [37]. Briefly, the animals were housed on a $12 \mathrm{~h}$ light/dark cycle with ad libitum access to food and water. After obtaining fresh spleen from male rat (250-300 g) under anesthesia, all connective tissues were removed from the spleen by pushing it through a $70 \mu \mathrm{m}$ mesh filter. The spleen tissue was then suspended in ice cold PBS and centrifuged at $400 \mathrm{~g}$ for $8 \mathrm{~min}$. After centrifugation, the supernatant was discarded, the tissue pellet was resuspended in $5 \mathrm{ml}$ of red blood cell lysis buffer (Sigma-Aldrich, MO, USA) and incubate on ice for 5 min. The sample was then diluted with $5 \mathrm{ml}$ of PBS and centrifuged at $400 \mathrm{~g}$ for $8 \mathrm{~min}$. The supernatant was again discarded and the reaming pellet was suspended in phenol-free RPMI with $10 \% \mathrm{FBS}$. After this, the sample was titrated multiple times and then was run through a $40 \mu \mathrm{m}$ mesh filter to remove clumps. The splenocytes count and viability was then checked via Trypan blue exclusion. For the in vitro assay, splenocytes $\left(2 \times 10^{6}\right.$ cells $\left./ \mathrm{ml}\right)$ were activated with lipopolysaccharides (LPS) or concanavalin A (ConA), alone or in the presence of HUCB cells (1:20; HUCB cells:splenocytes ratio) in a 96-well plates. LPS stimulates cells through TLR4, causing the release of inflammatory cytokines and upregulation of costimulatory molecules on antigen-presenting cells and ConA activates T cells. Both TLR4 and T-cell activation are linked to TBI. Culture supernatant was collected $24 \mathrm{~h}$ after LPS treatment or $72 \mathrm{~h}$ after ConA activation and the samples analyzed utilizing a TNF- $\alpha$ or IFN- $\gamma$ (Biolegend, CA, USA) and PGE2 (Cayman, MI, USA) ELISA kits following manufacturer's protocol.

\section{Real-time PCR}

Cells were seeded at $10^{6}$ cells per well in a 6-well plate. For each donor, the cells were grown with/without the presence of TNF- $\alpha(20 \mathrm{ng} / \mathrm{ml})$ or IFN- $\gamma(20 \mathrm{ng} / \mathrm{ml})$ for $24 \mathrm{~h}$. The following day, total RNA was extracted with QIAGEN RNAeasy Micro Kit. Reverse transcription of RNA was performed using Multiscribe DNA polymerase (Applied Biosystems), and Taqman Real-Time PCR (Applied Biosystems) was performed in $20 \mu \mathrm{l}$ reactions on an Applied Biosystems 7900 Real-Time PCR System. All procedures were conducted according to the manufacturer's instructions. List of primers used: GAPDH (Hs02758991_g1), IDO-1 (Hs00984148_m1), PTGS2 (COX-2, Hs00153133_m1), IL1RN(Hs00893626_m1) and TNFAIP6 (TSG-6, Hs01113602_m1), all from Applied Biosystems.

\section{Statistical analyses}

All data are represented as means \pm standard deviation. Comparisons between means of each group were made with the use of one-way analysis of variance followed by Tukey or Sidak's post hoc correction for multiple comparisons. Significance of correlations were determined by two-tailed Pearson's coefficients of correlation. All statistics were calculated using Graphpad Prism.

\section{Results}

HUCB cells delivered at $72 \mathrm{~h}$ postinjury effectively restore brain vascular integrity

In agreement with previous published reports on excellent cell viability/stability of cryopreserved HUCBs, cell viability in this study after thaw/preinjection was approximately $80 \%$ in all cord blood units. We then used a systemic dose of $25 \times 10^{6} \mathrm{TNC} / \mathrm{kg}$ body. We tested two different treatment schedules where HUCB cells from two different donors (CB-1 and CB-2) were systemically delivered at either 24 or $72 \mathrm{~h}$ postinjury with BBB integrity 


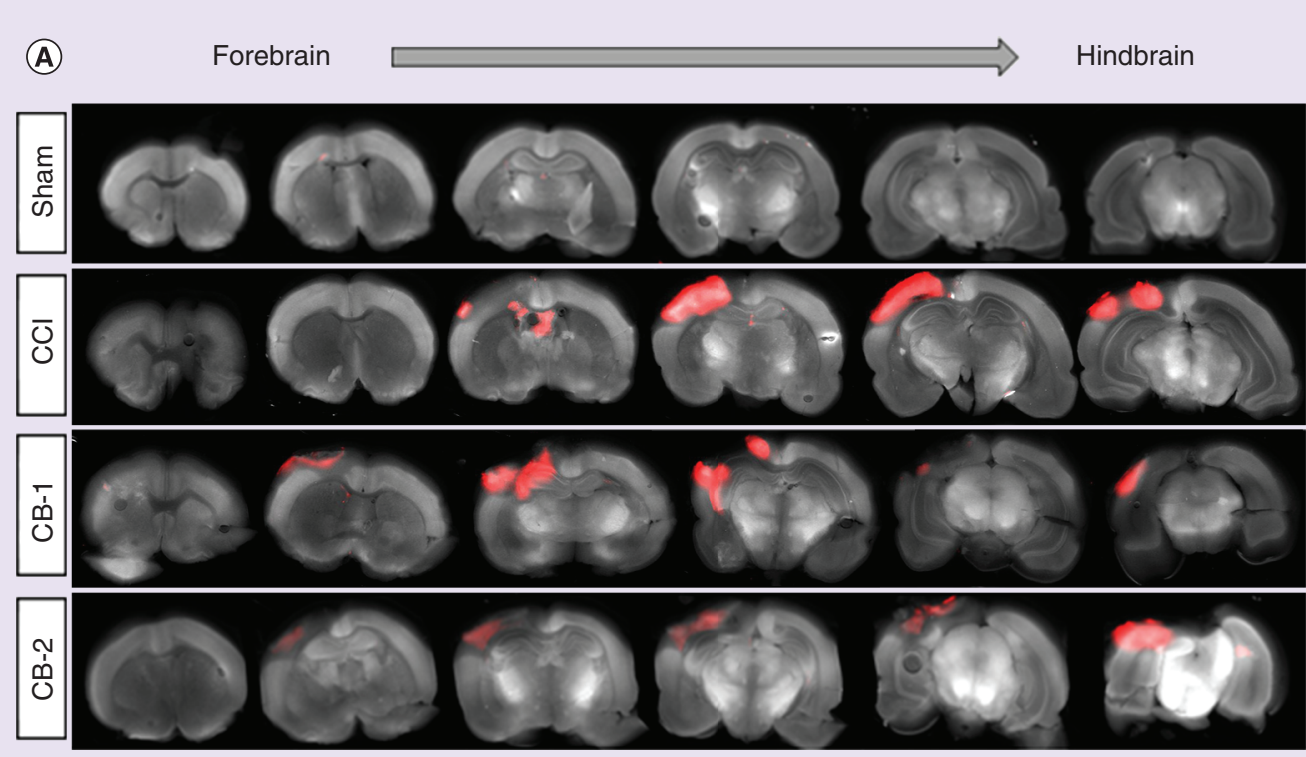

(B)
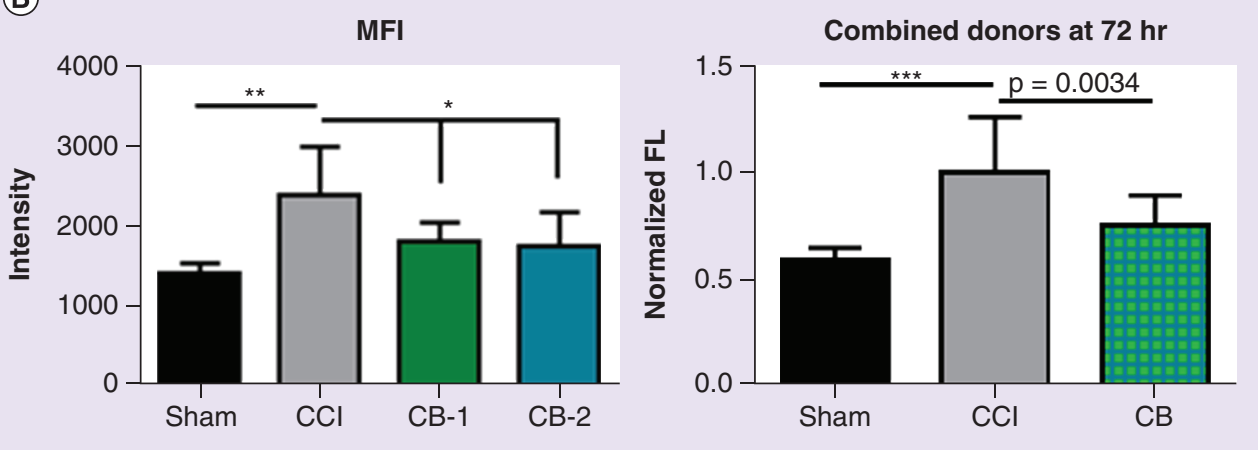

Figure 1. Dye extravasation following human umbilical cord blood treatment for traumatic brain injury. Rats were treated for a severe traumatic brain injury delivered by controlled cortical impact using $25 \times 10^{6}$ total nucleated cell/kg from two different donors (CB-1, CB-2) delivered at $72 \mathrm{~h}$ after injury. After $96 \mathrm{~h}$, respectively rats were injected intravenously with a far-red fluorescent dye-conjugated dextran, followed by perfusion. Blood-brain barrier permeability is then imaged using a laser scanner system (A) for quantitative analysis (B). ${ }^{*} \mathrm{p}<0.05 ;{ }^{* *} \mathrm{p}<0.01 ; * * * \mathrm{p}<0.001$.

MFI: Mean fluorescence intensity.

assessed at 72 or $96 \mathrm{~h}$, respectively. We observed that HUCB cells administered at $72 \mathrm{~h}$ after the injury resulted in a significant reduction in the mean fluorescence intensity of dye extravasation at $96 \mathrm{~h}$ postinjury $(\mathrm{p}<0.05)$. These results indicated a treatment effect as restoration of BBB integrity (Figure 1). On the other hand, we did not observe any treatment effect when cells were delivered $24 \mathrm{~h}$ postinjury (Supplementary Figure 1). Notably, treatment with HUCB cells administered $72 \mathrm{~h}$ postinjury also resulted in a significant decrease in the mean fluorescence intensity when grouped by the combined donors, combined treatment timing and the total effects of treatment (Figure 2).

\section{HUCB cells significantly suppress inflammatory responses in vitro}

We have previously used a cytokine inhibition assay to measure the immunomodulatory capacity of various therapeutic cell populations, wherein primary rat splenocytes are stimulated with either LPS or ConA, cocultured with the cells of interest, and the accumulated TNF- $\alpha$ or IFN- $\gamma$ is then assayed after either 24 or $48 \mathrm{~h}$, respectively [32,35-38]. Rat splenocytes are used as a reliable source of naive mixed leukocytes, simulating a systemic immune response by allowing for cross-talk between various immune cells. LPS treatment primarily stimulates monocytes and macrophages that rapidly respond by secreting copious amounts of TNF- $\alpha$ within hours that is readily detected $24 \mathrm{~h}$ later by ELISA. ConA preferentially binds to and activates T cells, resulting in the release of IFN- $\gamma$ that is 

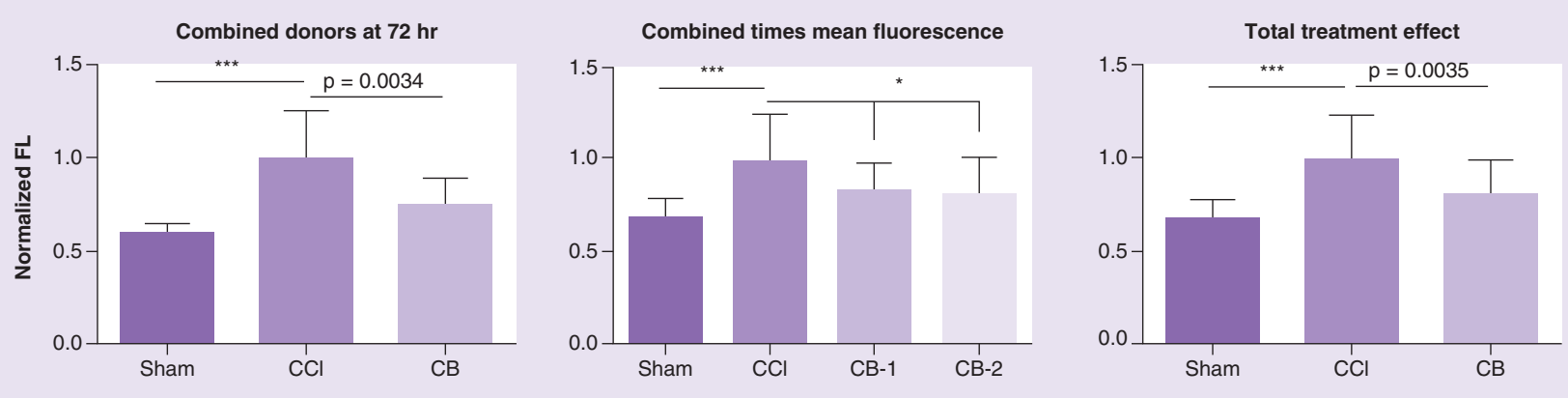

Figure 2. Cumulative treatment effects of human umbilical cord blood on blood-brain barrier. The results of the blood-brain barrier dye extravasation assay were pooled in various combinations to better assess the total treatment effect when human umbilical cord blood were delivered at $72 \mathrm{~h}$ after injury (left), pooled by the individual donors (middle) or compiled from all treatment groups and all treatment schedules tested (right).

$* p<0.05 ; * * * p<0.001$.

easily measured after 48-72 h in culture. In typical cocultures with mesenchymal stem cells (MSCs), we observe a significant decrease in TNF- $\alpha$ and IFN- $\gamma$ accumulation.

HUCB cells from CB-1 and CB-2 were added to LPS or ConA-stimulated splenocytes at ratios ranging from 1:10 to 1:80 HUCB:splenocytes and cultured for 24 or $48 \mathrm{~h}$, respectively. All HUCB donors and concentrations significantly decreased the accumulation of TNF- $\alpha$ compared with splenocytes alone, with CB-1 demonstrating a small-dose response (Figure 3A). We additionally measured the accumulation of $\mathrm{PGE}_{2}$ in the cocultures, as we have previously demonstrated that MSCs use $\mathrm{PGE}_{2}$ to modulate TNF- $\alpha$ in vitro and that MSCs require COX2 to decrease neuroinflammation when used to treat $\mathrm{CCI}$ in rats [32]. There was no significant change in $\mathrm{PGE}_{2}$ concentrations between control cultures and HUCB containing cultures (data not shown).

To address the possibility of donor variation and sampling error, we surveyed three additional HUCB donors (CB-3, CB-4 and CB-5). Cells from all three HUCB units demonstrated a dose-response curve that significantly decreased TNF- $\alpha$ at 1:20 and 1:40 HUCB:splenocyte ratios (Figure 3B). We also observed a significant decrease in IFN- $\gamma$ accumulation after stimulation with ConA in at least the 1:20 ratio with all three donors, with indications of a dose-response (Figure 3B).

The potential role of $\mathrm{PGE}_{2}$ in the observed cytokine inhibition was assayed by ELISA from the LPS-stimulated cocultures after $24 \mathrm{~h}$ (Figure 4). None of the cocultures demonstrated a significant change in the accumulation of $\mathrm{PGE}_{2}$ compared with the control splenocytes; however, the 1:20 dilution consistently demonstrated higher amounts of $\mathrm{PGE}_{2}$ compared with the other dilutions tested, creating the appearance of a dose response where more HUCB in the cocultures resulted in an increase in $\mathrm{PGE}_{2}$.

To further investigate possible mechanisms of immune modulation by HUCB, we performed quantitative PCR analysis of PTSG (COX2), IDO and TSG-6 in either normal cultures or after being exposed to TNF- $\alpha$ or IFN- $\gamma$ to partially simulate the coculture conditions (Figure 4). In the control cultures, all three donors had comparable expression of all three genes, with a maximum difference of 2.5 -fold difference in expression (measured as $\Delta \Delta \mathrm{Ct}$ relative to GAPDH and CB-5 control cells). Beyond this initial comparison, the three donors demonstrated differing gene expression patterns in response to the cytokines for the three genes assayed with one exception: all three donors demonstrated an increase in TSG-6 expression when stimulated with TNF- $\alpha$, as would be expected based upon the name, 'TNF- $\alpha$ Stimulated Gene 6.'

The immunomodulatory potential of HUCB cells correlates with the expression of COX2 \& IDO In an attempt to decipher the relationship between the expression of COX2,IDO,TSG-6, $\mathrm{PGE}_{2}$ and antiinflammatory activity of HUCB cells in the activated splenocyte cocultures, we used correlational analysis to further parse the results of the various assays (Supplementary Figures 2-4). We have grouped the correlating plots according to gene expression, with $\mathrm{R}^{2}$ values higher than 0.8 surrounded by a red box. The resulting data, while limited to a small data set, present several likely conclusions.

First, expression of PTSG (COX2) directly correlates with the ability of HUCB to decrease TNF- $\alpha$, resulting in a decrease in accumulated TNF- $\alpha$, but only when HUCB are exposed to TNF- $\alpha$ (Figure 5). This is consistent 


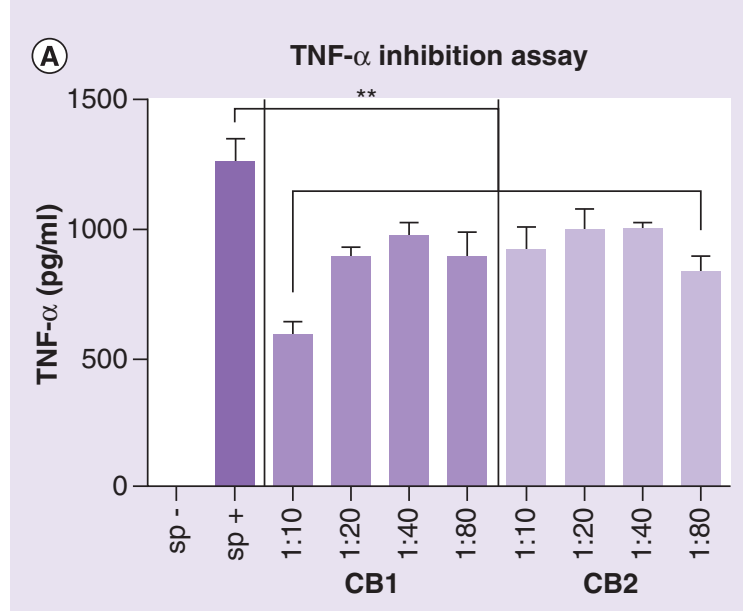

(B)

TNF- $\alpha$ inhibition assay

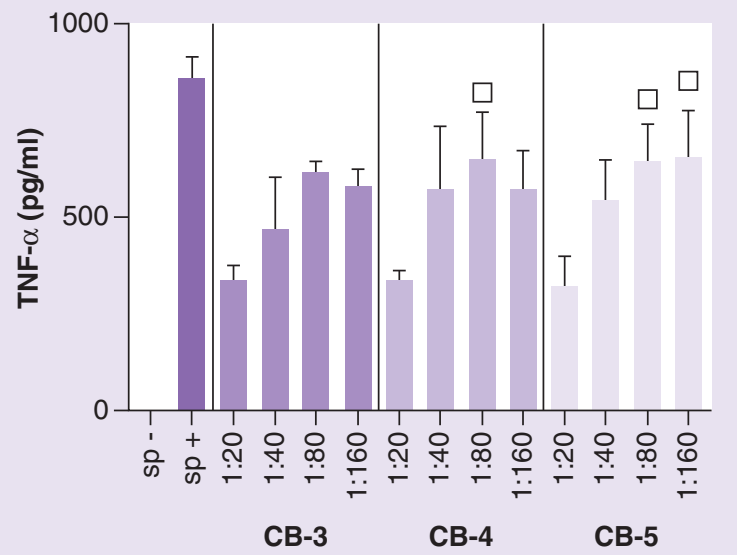

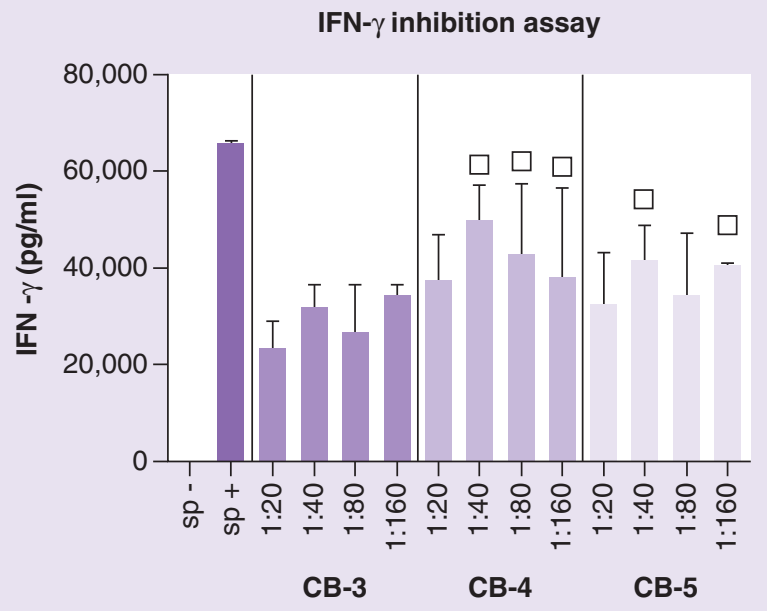

Figure 3. Human umbilical cord blood inhibits the release of TNF- $\alpha$ from activated splenocytes. Primary rat splenocytes were isolated fresh and activated with either lipopolysaccharides or concanavalin A immediately prior to the addition of human umbilical cord blood to culture. The resulting release of TNF- $\alpha$ and IFN- $\gamma$ was then assayed by ELISA after 24 or $48 \mathrm{~h}$ in cultures (respectively). (A) Human umbilical cord blood significantly reduced the amount of TNF- $\alpha$ released by lipopolysaccharides-activated splenocytes. (B) Three additional donors were used to assay donor variability, with all three demonstrating a consistent dose response curve by TNF- $\alpha$ inhibition and IFN- $\gamma$ to a lesser extent. All values are statistically different than positive control unless noted ( $\square=$ not statistically significant compared with positive control).

$* * \mathrm{p}<0.01$.

$\mathrm{sp}^{-}$: Splenocyte only negative control; $\mathrm{sp}^{+}$: Activated splenocyte only positive control.

with our previous work with other cell therapies, including MSC, and indicates a negative feedback or homeostatic response. Similarly, the pretreatment of HUCB with IFN- $\gamma$ resulted in a direct correlation between PTSG (COX2) and IFN- $\gamma$ inhibition (Figure 5). Surprisingly, both TNF- $\alpha$ and IFN- $\gamma$ treatments on HUCB resulted in a negative correlation between expression of PTSG (COX2) and accumulated $\mathrm{PGE}_{2}$.

The relationship between the expression of IDO and HUCB activity contained additional interesting results. Namely, IDO expression demonstrated a similar correlation with HUCB performance in the activated cocultures, but under the opposite condition. Specifically, IDO induction by IFN- $\gamma$ exposure correlates with TNF- $\alpha$ inhibition, while IDO induction by TNF- $\alpha$ exposure correlates with IFN- $\gamma$ inhibition (Figure 5).

Finally, we examined $T S G-6$ expression as it relates to immunomodulation and $\mathrm{PGE}_{2}$ concentration. Interestingly, while there is a possible relationship between TSG- 6 induction by TNF- $\alpha$ and the ability to inhibit IFN- $\gamma$, there is a much stronger direct correlation between the expression of TSG- 6 in response to TNF- $\alpha$ and IFN- $\gamma$ stimulation 

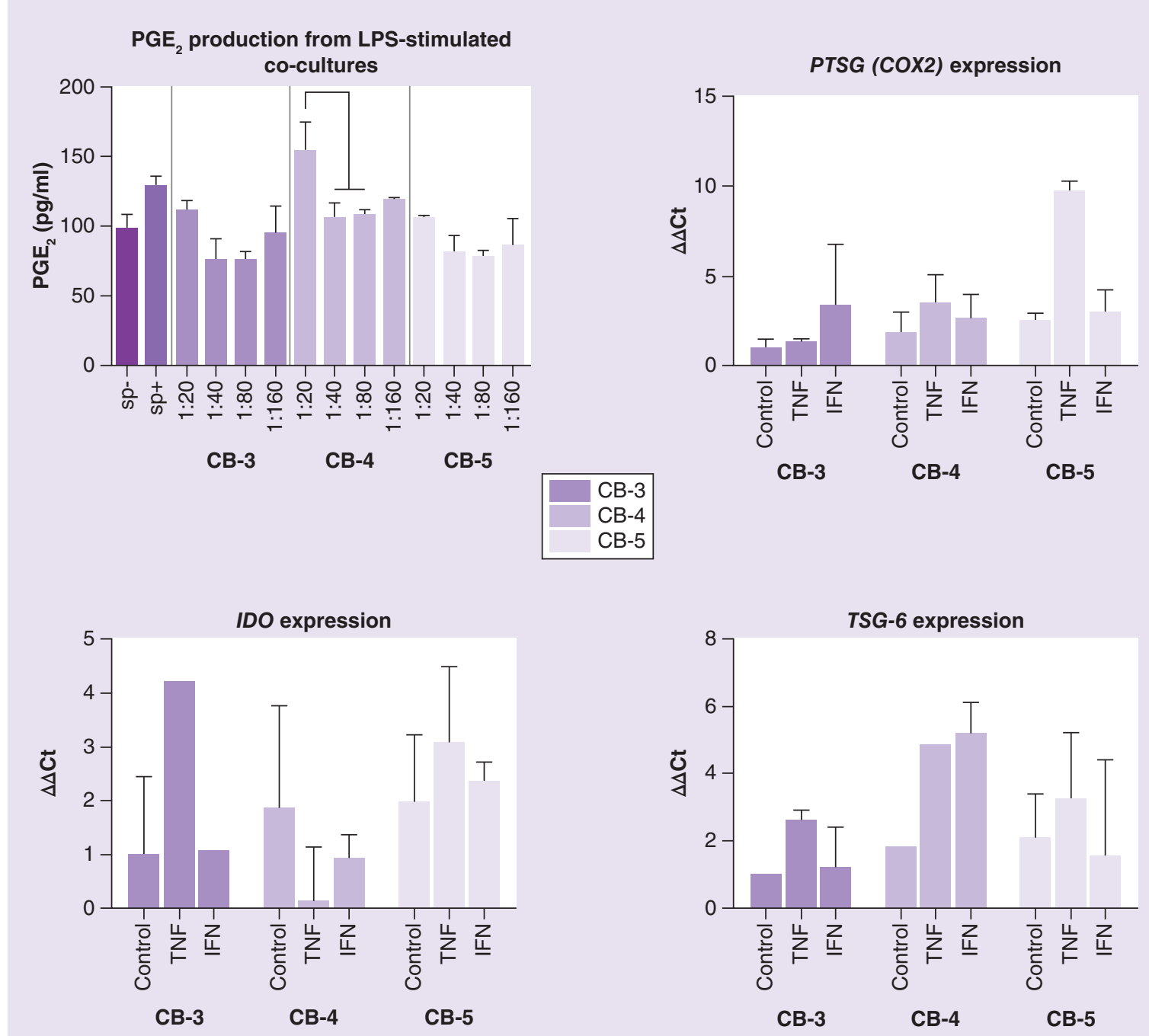

Figure 4. Potential mechanisms of immunomodulation by human umbilical cord blood. The effects of human umbilical cord blood on $\mathrm{PGE}_{2}$ concentrations were assayed from the lipopolysaccharides-stimulated cocultures (top left). Quantitative real-time PCR was used to evaluate expression of PTSG (COX2) from normal and TNF- $\alpha$ or IFN- $\gamma$ exposed human umbilical cord blood (top right), IDO (bottom left) and TSG-6 (TNFAIP6; bottom right), resulting in changes in expression (presented as delta-delta Ct or $\triangle \triangle \mathrm{Ct}$ relative to GAPDH and CB-3 control cells).

and the resulting measured concentration of $\mathrm{PGE}_{2}$ in the cocultures, where increasing amounts of TSG-6 expression indicate increasing amounts of $\mathrm{PGE}_{2}$ (Figure 5).

\section{Discussion}

In this study, we demonstrated the efficacy of umbilical cord blood cells from different donors on BBB integrity in experimental TBI and reported the immunomodulatory abilities of HUCB cells in vitro. BBB damage is a hallmark of TBI pathology [39]. Parenchymal and vascular damages due to mechanical injury to the brain lead to BBB breakdown. Once this barrier is disrupted, secondary pathologies such as edema, inflammation and neuronal hyperexcitability occur. These changes further trigger a cascade of additional pathomechanism that leads to perpetual damage of neurons [40]. Several studies have utilized HUCB cells to treat TBI and other CNS injuries [21,41-44]. However, these studies were mainly focused on anti-inflammatory and neurogenesis/neuroprotective abilities of cord blood cells. To best of our knowledge, there is no study that has demonstrated the effect of HUCB cells on TBI-associated neurovascular pathology. We used the CCI rat model in this study because a concomitant alteration in BBB following CCI in animals is well documented [32,35]. 

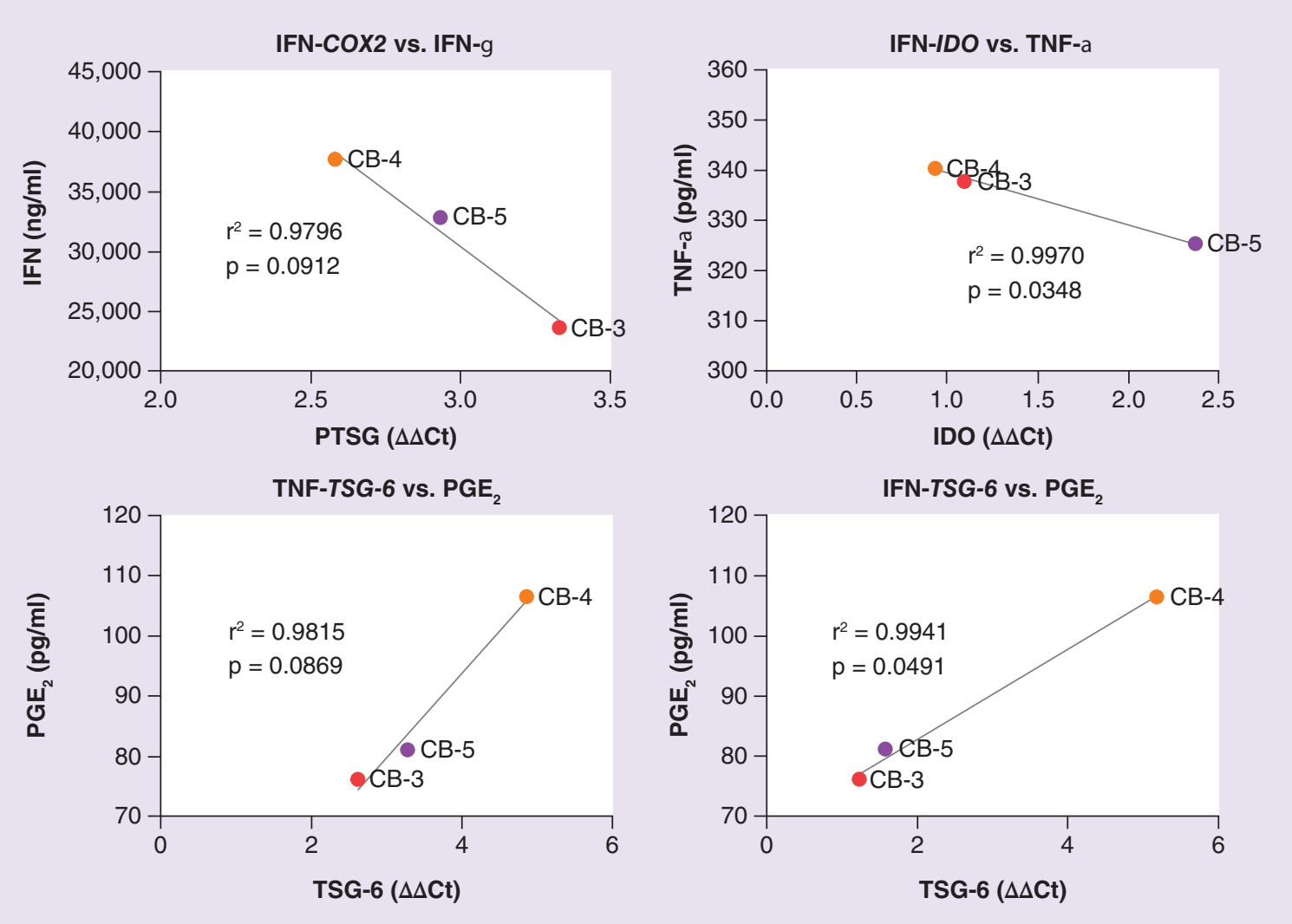

Figure 5. Correlational analysis between data sets. The ability of human umbilical cord blood to inhibit IFN- $\gamma$, inhibit TNF- $\alpha$ in an activated splenocyte assay (Figure 3) or produce PGE 2 (Figure 4) was correlated with the expression of COX2, IDO and TSG- 6 after stimulating human umbilical cord blood with either IFN- $\gamma$ or TNF- $\alpha$ (Figure 4). The resulting correlations were evaluated for significance using a two-tailed Pearson coefficient.

HUCB: Human umbilical cord blood.

In this study, we observed that systemic infusions of HUCB cells improved brain vascular integrity by reducing the BBB leakage in CCI animals (Figure 1). We chose intravenous administration as the route for cell because it is less invasive and clinically relevant. A previous study has also reported that intravenous administration of HUCB cells was at least as effective and/or more effective than direct intracerebral cell transplant in a stroke animal model [45]. We infused $2.5 \times 10^{7} \mathrm{HUCB}$ cells/ $/ \mathrm{kg}$ bodyweight for the treatment. In an unpublished pilot study, a lower cell dose $\left(9 \times 10^{6} \mathrm{HUCB}\right.$ mononuclear cells/kg bodyweight $)$ at $24 \mathrm{~h}$ postinjury in CCI rats failed to show any effect on BBB permeability at $72 \mathrm{~h}$ after the injury (data not shown). Based on our pilot data, available literature and current clinical trial designs using HUCB cells, the dose of this study was determined $[27,28,46]$. We observed no mortality and absence of any major sign of distress in CCI rats after the intravenous infusion of cells and during the course of study.

As BBB permeability is a dynamic process that changes as a function of time after injury, there were a number of considerations in designing our primary experiment. Several studies have reported a biphasic pattern of BBB disruption after experimental TBI. Two peak phases of BBB leakage were reported to be within $24 \mathrm{~h}$ of injury and then again at $48-72 \mathrm{~h}$ postinjury $[31,47,48]$. We used our previous experiences $[32,35,49]$ and the reports from other group to select a time point to evaluate the BBB where the cell therapy would have sufficient time to elicit an effect while still being able to detect significant changes in BBB permeability, which becomes increasingly difficult more than $96 \mathrm{~h}$ after injury. We found that HUCB cells infused $72 \mathrm{~h}$ after the injury were able to significantly decrease $(\mathrm{p}<0.05)$ the BBB permeability at $96 \mathrm{~h}$ postinjury (Figure 1), however, no therapeutic effect was observed in the CCI rats that received cells $24 \mathrm{~h}$ after the injury (Supplementary Figure 1). Conventional wisdom has been that earlier cell delivery, irrespective of mechanism of action, would be better and the majority of investigators have indeed opted to administer cells in the acute phase of the injury (within $24 \mathrm{~h}$ after TBI) [50]. However, 
some investigators have also reported significant recovery after delivering stem cells as late as up to 1 week postTBI [51,52]. Although further investigation is required to demonstrate mechanism of action of HUCB at different time points postinjury, it is possible that the acute inflammation and hostile edematous CNS microenvironment within $24 \mathrm{~h}$ of injury [53,54] may not be very conducive for infused cells to exert any therapeutic effect at early time point. Nevertheless, the present study demonstrates that systemic infusion of HUCB cells in CCI rats is not only well-tolerated but also improves brain vasculature integrity.

Another major repercussion of traumatic injury in the brain is an ensuing neuroinflammation [55]. In the event of brain trauma, BBB breakdown permits infiltration of peripheral immune cells into the CNS leading to edema formation and neuroinflammation [31]. This neuroinflammation in return further affect vascular permeability. HUCB cells have shown to effectively modulate immune and inflammatory responses in different neurological and immunological conditions. However, not much information is available on underlying molecular mechanism by which these cells modulate the immune response. We demonstrated HUCB cells were able to significantly reduce the amount of TNF- $\alpha$ and IFN- $\gamma$ released by activated primary rat splenocytes in vitro (Figure 3A \& B). We used the activated splenocytes assays because of the inherent advantage of this assay in monitoring immunomodulatory potential of therapeutic cells. Splenocytes contain an unrefined mixture of leukocytes capable of creating incredibly complex signaling pathways in the context of an inflammatory response. In this study, the demonstration of HUCB-mediated immunomodulation of global inflammation is valuable in understanding the potency of these cells to treat neuroinflammatory condition like TBI.

It is important to note that HUCB contains a mix of cell subsets, including endothelial progenitor cells, MSCs, Tregs and suppressive monocytes. Although it is not very well understood how each of the different UCB-derived cells contributes to overall efficacy, it is possible that the therapeutic benefits observed in this study could be attributed to different HUCB cell populations. Previous studies have shown a negative linear correlation between $\mathrm{CD}_{3} 4^{+}$cell and BBB permeability in TBI [56] and endothelial progenitor cells, Tregs and monocytes in HUCB were found to modulate the peripheral and central immune response [57].

Our intent in this work was primarily to investigate the efficacy of HUCB to treat TBI using BBB as a biomarker for therapeutic activity. As a secondary goal, we sought to address several possible mechanisms of immunomodulation using our experience in the use of MSC and recent reports in the literature to assemble a short list of possible candidate mechanisms, including $C O X 2 / \mathrm{PGE}_{2}, I D O$ and TSG-6. We observed that COX-2, IDO, TSG-6 and $\mathrm{PGE}_{2}$ all have a role in the mechanisms by which HUCB modulate TNF- $\alpha$ and IFN- $\gamma$ by virtue of correlations. An important note is that any conclusion based upon correlational analysis performed with this limited data set may be premature, additional donors and replicates would further strengthen the putative relationships between gene expression, gene induction, $\mathrm{PGE}_{2}$ secretion and immunomodulatory action. A lack of statistical significance in some comparisons due to a limited sample size in this study may partially be ameliorated by existing strong literature support in parallel studies using subpopulations of HUCB [26,58,59]. Another limitation of this study is that the candidate mechanisms investigated are far from comprehensive, and additional targets would likely be implicated by a more thorough evaluation of the transcriptome, secretome and proteome. The study was limited by technical obstacles involved with working with HUCB cells, such as the finite supply of cells from each donor, assuming the cord blood units are not expanded ex vivo and the intent to utilize the cells in a timely fashion with respect to the initial injury thereby precluding subculture. It is likely that there are many dynamic mechanisms of action by which HUCB cells can modulate inflammation as both a contributor and modulator of an immune response. This may include direct activity through traditional pro- and anti-inflammatory cytokines, as well as autocrine and paracrine activity through growth factors, metabolites and contact-mediated mechanisms.

In our previous work, we were able to demonstrate a strong predictive link between MSC expression of COX2 and production of $\mathrm{PGE}_{2}$ [32]. While COX2/PGE 2 is involved in decreasing both TNF- $\alpha$ and IFN- $\gamma$ in the activated splenocytes in this study, it is likely one component of a larger response that is specific to each cytokine. Inverse relationship between PTSG and PGE2 indicates the presence of a confounding or masking condition. This could be due to the simplicity of using TNF- $\alpha / \mathrm{IFN}-\gamma$ alone to model an inflamed system. The complementary role of PTSG/COX2 and IDO is indicated, whereby TNF- $\alpha$ treatment results in correlation between COX2 and TNF- $\alpha$ inhibition and IDO and IFN- $\gamma$ activity, while IFN- $\gamma$ treatment results in the opposite. This observation positions $I D O$ as being a complementary mechanism, whereby HUCB cells may be able to further regulate inflammatory cascades. Additional mechanistic studies are required to fully understand the relationship between these genes, cell therapy and immunomodulatory activity. TSG-6/PGE2 relationship in this study indicates that TSG-6 may be coexpressed with PGE2. Interestingly, comparing the expression of the three genes in each condition results in 
only positive correlations in the control cultures (data not shown). PTSG and TSG-6 have an inverse relationship in expression when induced by IFN- $\gamma$, while IDO and TSG- 6 have inverse correlation after treatment with TNF$\alpha$. No correlations were detected between PTSG and IDO. Similarly, despite colloquial observations, no strong correlations were detected between PGE2, TNF- $\alpha$ inhibition and IFN- $\gamma$ inhibition at any of the HUCB:splenocyte concentrations tested. Additional work is required to fully establish the natural range of donor variability and the possible effects of real-world handling on HUCB biology and therapeutic potential.

\section{Conclusion}

Standardized protocols to manage severe TBI are multifactorial and include maintenance and/or support of cerebral perfusion pressure and intracranial pressure. Although these practices can stabilize a patient who is already injured, they are incapable of reversing primary or secondary injury. The future of TBI care lies in development of therapies focused on limiting secondary injuries and enhanced repair of brain. This preclinical study indicates that HUCB cells have potential to repair BBB and ammoniate neuroinflammation in TBI. These results indicate that HUCB cells may be useful and feasible for treatment of brain injuries.

\section{Translational perspective}

HUCB cells have a long history of clinical use and a well-understood human safety profile, making this cell therapy highly useful to treat traumatic brain injury with an accelerated translational pathway to clinical trials.

\section{Data availability}

The primary data supporting this work are not publicly available due to terms of the sponsored research agreement between the University of Texas Health Science System and Cbr Systems, Inc. However, data may be made available upon reasonable request from the corresponding author with the consent of Cbr Systems, Inc.

\section{Summary points}

- Human umbilical cord blood (HUCB) cells have pleiotropic effects in traumatic brain injury.

- Systemic single-dose infusion of HUCB cells significantly restored blood-brain integrity in experimental traumatic brain injury.

- HUCB cells have shown strong immunomodulatory abilities in vitro.

\section{Supplementary data}

To view the supplementary data that accompany this paper please visit the journal website at: www.futuremedicine.com/doi/full/ 10.2217/rme-2018-0106

\section{Financial \& competing interests disclosure}

KS Brown, ML Skiles and HL Brown receive payment as employees of Cbr Systems, Inc. (Cord Blood Registry ${ }^{\circledR}$ ). Dr. Charles S. Cox, Jr serves on the Scientific and Medical Advisory Board of Cbr Systems, Inc. This study was partially funded through a sponsored research agreement between the University of Texas Health Science Center at Houston and Cbr Systems, Inc. and through the generous support of the Clare A. Glassell Family Pediatric Stem Cell Research Fund. The authors have no other relevant affiliations or financial involvement with any organization or entity with a financial interest in or financial conflict with the subject matter or materials discussed in the manuscript apart from those disclosed.

No writing assistance was utilized in the production of this manuscript.

\section{Ethical conduct of research}

The study design and animal usage were reviewed and approved by the University of Texas Health Science Center at Houston Institutional Animal Care and Use Committee for compliance with all applicable regulations and welfare laws and policies. All animal procedures and housing were in compliance with the USDA Animal Welfare Act, the Guide for the Care and Use of Laboratory Animals, the Guide for the Care and Use of Agricultural Animals in Research and Teaching, Public Health Service National Institutes of Health Office of Laboratory Animal Welfare and the American Veterinary Medical Association Guidelines for Euthanasia. 
Open access

This work is licensed under the Attribution-NonCommercial-NoDerivatives 4.0 Unported License. To view a copy of this license, visit http://creativecommons.org/licenses/by-nc-nd/4.0/

\section{References}

1. Dekosky ST, Blennow K, Ikonomovic MD, Gandy S. Acute and chronic traumatic encephalopathies: pathogenesis and biomarkers. Nat. Rev. Neurol. 9(4), 192-200 (2013).

2. Maas AI, Stocchetti N, Bullock R. Moderate and severe traumatic brain injury in adults. Lancet Neurol. 7(8), 728-741 (2008).

3. Jackson ML, Srivastava AK, Cox CS Jr. Preclinical progenitor cell therapy in traumatic brain injury: a meta-analysis. J. Surg. Res. 214, 38-48 (2017)

4. Cox CS Jr, Hetz RA, Liao GP et al. Treatment of severe adult traumatic brain injury using bone marrow mononuclear cells. Stem Cells 35(4), 1065-1079 (2017).

5. Savitz SI, Cox CS Jr. Concise review: cell therapies for stroke and traumatic brain injury: targeting microglia. Stem Cells 34(3), 537-542 (2016).

6. Liao GP, Harting MT, Hetz RA et al. Autologous bone marrow mononuclear cells reduce therapeutic intensity for severe traumatic brain injury in children. Pediatr. Crit. Care Med. 16(3), 245-255 (2015).

7. Tajiri N, Acosta SA, Shahaduzzaman M et al. Intravenous transplants of human adipose-derived stem cell protect the brain from traumatic brain injury-induced neurodegeneration and motor and cognitive impairments: cell graft biodistribution and soluble factors in young and aged rats. J. Neurosci. 34(1), 313-326 (2014).

8. Cox CS Jr, Baumgartner JE, Harting MT et al. Autologous bone marrow mononuclear cell therapy for severe traumatic brain injury in children. Neurosurgery 68(3), 588-600 (2011).

9. Walker PA, Harting MT, Jimenez F et al. Direct intrathecal implantation of mesenchymal stromal cells leads to enhanced neuroprotection via an NFkappaB-mediated increase in interleukin-6 production. Stem Cells Dev. 19(6), 867-876 (2010).

10. Koliatsos VE, Xu L, Cummings BJ. Stem cell therapies for traumatic brain injury. Regen. Med. 10(8), 917-920 (2015).

11. Ballen KK, Gluckman E, Broxmeyer HE. Umbilical cord blood transplantation: the first 25 years and beyond. Blood 122(4), 491-498 (2013).

12. Ballen K. Umbilical cord blood transplantation: challenges and future directions. Stem Cells Transl. Med. 6(5), 1312-1315 (2017).

13. Mcguckin CP, Forraz N. Potential for access to embryonic-like cells from human umbilical cord blood. Cell Prolif. 41(Suppl. 1), 31-40 (2008).

14. Pimentel-Coelho PM, Rosado-De-Castro PH, Da Fonseca LM, Mendez-Otero R. Umbilical cord blood mononuclear cell transplantation for neonatal hypoxic-ischemic encephalopathy. Pediatr. Res. 71(4 Pt 2), 464-473 (2012).

15. Cho PS, Messina DJ, Hirsh EL et al. Immunogenicity of umbilical cord tissue derived cells. Blood 111(1), 430-438 (2008).

16. Nikiforow S, Li S, Snow K et al. Lack of impact of umbilical cord blood unit processing techniques on clinical outcomes in adult double cord blood transplant recipients. Cytotherapy 19(2), 272-284 (2017).

17. Wagner JE, Barker JN, Defor TE et al. Transplantation of unrelated donor umbilical cord blood in 102 patients with malignant and nonmalignant diseases: influence of CD34 cell dose and HLA disparity on treatment-related mortality and survival. Blood 100(5), $1611-1618$ (2002)

18. Barker JN, Scaradavou A, Stevens CE. Combined effect of total nucleated cell dose and HLA match on transplantation outcome in 1061 cord blood recipients with hematologic malignancies. Blood 115(9), 1843-1849 (2010).

19. Sanberg PR, Willing AE, Garbuzova-Davis S et al. Umbilical cord blood-derived stem cells and brain repair. Ann. NY Acad. Sci. 1049, 67-83 (2005).

20. Newman MB, Davis CD, Kuzmin-Nichols N, Sanberg PR. Human umbilical cord blood (HUCB) cells for central nervous system repair. Neurotox. Res. 5(5), 355-368 (2003).

21. Lu D, Sanberg PR, Mahmood A et al. Intravenous administration of human umbilical cord blood reduces neurological deficit in the rat after traumatic brain injury. Cell Transplant. 11(3), 275-281 (2002).

22. Chen J, Sanberg PR, Li Y et al. Intravenous administration of human umbilical cord blood reduces behavioral deficits after stroke in rats. Stroke 32(11), 2682-2688 (2001).

23. Damien P, Allan DS. Regenerative therapy and immune modulation using umbilical cord blood-derived cells. Biol. Blood Marrow Transplant. 21(9), 1545-1554 (2015).

24. Shawki S, Gaafar T, Erfan H, El Khateeb E, El Sheikhah A, El Hawary R. Immunomodulatory effects of umbilical cord-derived mesenchymal stem cells. Microbiol. Immunol. 59(6), 348-356 (2015).

25. Sydserff S, Sutton EJ, Song D et al. Selective alpha7 nicotinic receptor activation by AZD0328 enhances cortical dopamine release and improves learning and attentional processes. Biochem. Pharmacol. 78(7), 880-888 (2009). 
26. Vendrame M, Gemma C, De Mesquita D et al. Anti-inflammatory effects of human cord blood cells in a rat model of stroke. Stem Cells Dev. 14(5), 595-604 (2005).

27. Sun JM, Song AW, Case LE et al. Effect of autologous cord blood infusion on motor function and brain connectivity in young children with cerebral palsy: a randomized, placebo-controlled trial. Stem Cells Transl. Med. 6(12), 2071-2078 (2017).

28. Cotten CM, Murtha AP, Goldberg RN et al. Feasibility of autologous cord blood cells for infants with hypoxic-ischemic encephalopathy. J. Pediatr. 164(5), 973-979, e971 (2014).

29. Royo NC, Wahl F, Stutzmann JM. Kinetics of polymorphonuclear neutrophil infiltration after a traumatic brain injury in rat. Neuroreport 10(6), 1363-1367 (1999).

30. Soares HD, Hicks RR, Smith D, Mcintosh TK. Inflammatory leukocytic recruitment and diffuse neuronal degeneration are separate pathological processes resulting from traumatic brain injury. J. Neurosci. 15(12), 8223-8233 (1995).

31. Cunningham TL, Cartagena CM, Lu XC et al. Correlations between blood-brain barrier disruption and neuroinflammation in an experimental model of penetrating ballistic-like brain injury. J. Neurotrauma. 31(5), 505-514 (2014).

32. Kota DJ, Prabhakara KS, Toledano-Furman N et al. Prostaglandin E2 indicates therapeutic efficacy of mesenchymal stem cells in experimental traumatic brain injury. Stem Cells 35(5), 1416-1430 (2017).

33. Osier ND, Dixon CE. The controlled cortical impact model: applications, considerations for researchers, and future directions. Front. Neurol. 7, 134 (2016).

34. Liao GP, Olson SD, Kota DJ et al. Far-red tracer analysis of traumatic cerebrovascular permeability. J. Surg. Res. 190(2), 628-633 (2014).

35. Kota DJ, Prabhakara KS, Van Brummen AJ et al. Propranolol and mesenchymal stromal cells combine to treat traumatic brain injury. Stem Cells Transl. Med. 5(1), 33-44 (2016).

36. Diaz MF, Vaidya AB, Evans SM et al. Biomechanical forces promote immune regulatory function of bone marrow mesenchymal stromal cells. Stem Cells 35(5), 1259-1272 (2017).

37. Harting MT, Srivastava AK, Zhaorigetu $S$ et al. Inflammation-stimulated mesenchymal stromal cell-derived extracellular vesicles attenuate inflammation. Stem Cells doi:10.1002/stem.2730 (2017) (Epub ahead of print).

38. Kota DJ, Dicarlo B, Hetz RA, Smith P, Cox CS Jr, Olson SD. Differential MSC activation leads to distinct mononuclear leukocyte binding mechanisms. Sci. Rep. 4, 4565 (2014).

39. Alluri H, Wiggins-Dohlvik K, Davis ML, Huang JH, Tharakan B. Blood-brain barrier dysfunction following traumatic brain injury. Metab. Brain Dis. 30(5), 1093-1104 (2015).

40. Prins M, Greco T, Alexander D, Giza CC. The pathophysiology of traumatic brain injury at a glance. Dis. Model Mech. 6(6), 1307-1315 (2013).

41. Nan Z, Grande A, Sanberg CD, Sanberg PR, Low WC. Infusion of human umbilical cord blood ameliorates neurologic deficits in rats with hemorrhagic brain injury. Ann. NY Acad. Sci. 1049, 84-96 (2005).

42. De La Pena I, Sanberg PR, Acosta S, Lin SZ, Borlongan CV. Umbilical cord blood cell and granulocyte-colony stimulating factor: combination therapy for traumatic brain injury. Regen. Med. 9(4), 409-412 (2014).

43. Vyklicky L Jr, Benveniste M, Mayer ML. Modulation of N-methyl-D-aspartic acid receptor desensitization by glycine in mouse cultured hippocampal neurones. J. Physiol. 428, 313-331 (1990).

44. Chen $\mathrm{SH}$, Wang JJ, Chen $\mathrm{CH}$ et al. Umbilical cord blood-derived CD34(+) cells improve outcomes of traumatic brain injury in rats by stimulating angiogenesis and neurogenesis. Cell Transplant. 23(8), 959-979 (2014).

45. Willing AE, Lixian J, Milliken $\mathrm{M}$ et al. Intravenous versus intrastriatal cord blood administration in a rodent model of stroke. J. Neurosci. Res. 73(3), 296-307 (2003).

46. Dawson G, Sun JM, Davlantis KS et al. Autologous cord blood infusions are safe and feasible in young children with autism spectrum disorder: results of a single-center Phase I open-label trial. Stem Cells Transl. Med. 6(5), 1332-1339 (2017).

47. Baskaya MK, Rao AM, Dogan A, Donaldson D, Dempsey RJ. The biphasic opening of the blood-brain barrier in the cortex and hippocampus after traumatic brain injury in rats. Neurosci. Lett. 226(1), 33-36 (1997).

48. Tomkins O, Feintuch A, Benifla M, Cohen A, Friedman A, Shelef I. Blood-brain barrier breakdown following traumatic brain injury: a possible role in posttraumatic epilepsy. Cardiovasc. Psychiatry Neurol. 2011, 765923 (2011).

49. Prabhakara KS, Kota DJ, Jones GH, Srivastava AK, Cox CS Jr, Olson SD. Teriflunomide modulates vascular permeability and microglial activation after experimental traumatic brain injury. Mol. Ther. doi:10.1016/j.ymthe.2018.06.022 (2018) (Epub ahead of print).

50. Gennai S, Monsel A, Hao Q et al. Cell-based therapy for traumatic brain injury. Br. J. Anaesth. 115(2), 203-212 (2015).

51. Mahmood A, Lu D, Qu C, Goussev A, Chopp M. Long-term recovery after bone marrow stromal cell treatment of traumatic brain injury in rats. J. Neurosurg. 104(2), 272-277 (2006).

52. Shear DA, Tate MC, Archer DR et al. Neural progenitor cell transplants promote long-term functional recovery after traumatic brain injury. Brain Res. 1026(1), 11-22 (2004). 
53. Wilcockson DC, Campbell SJ, Anthony DC, Perry VH. The systemic and local acute phase response following acute brain injury. J. Cereb. Blood Flow Metab. 22(3), 318-326 (2002).

54. Thelin EP, Tajsic T, Zeiler FA et al. Monitoring the neuroinflammatory response following acute brain injury. Front. Neurol. 8, 351 (2017).

55. Jassam YN, Izzy S, Whalen M, Mcgavern DB, El Khoury J. Neuroimmunology of traumatic brain injury: time for a paradigm shift. Neuron 95(6), 1246-1265 (2017).

56. Jin X, Wang F, Liu X et al. Negative correlation of CD34+ cells with blood-brain barrier permeability following traumatic brain injury in a rat model. Microcirculation 21(8), 696-702 (2014).

57. Mcdonald CA, Penny TR, Paton MCB et al. Effects of umbilical cord blood cells, and subtypes, to reduce neuroinflammation following perinatal hypoxic-ischemic brain injury. J. Neuroinflammation 15(1), 47 (2018).

58. Seghatoleslam M, Jalali M, Nikravesh MR, Hosseini M, Hamidi Alamdari D, Fazel A. Therapeutic benefit of intravenous administration of human umbilical cord blood-mononuclear cells following intracerebral hemorrhage in rat. Iran J. Basic Med. Sci. 15(3), 860-872 (2012).

59. Badner A, Vawda R, Laliberte A et al. Early intravenous delivery of human brain stromal cells modulates systemic inflammation and leads to vasoprotection in traumatic spinal cord injury. Stem Cells Transl. Med. 5(8), 991-1003 (2016). 